NASA TECHNICAL MEMORANDU M

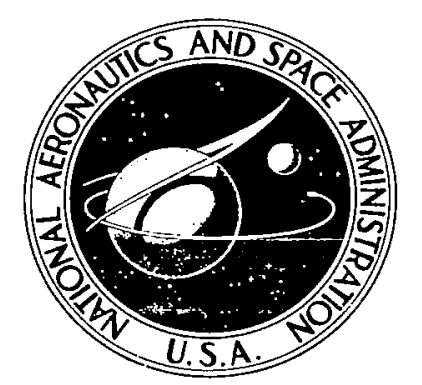

$\frac{\text { NASA TM }}{6.1} \frac{X-2024}{1}$

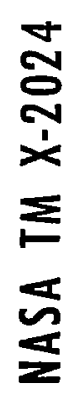

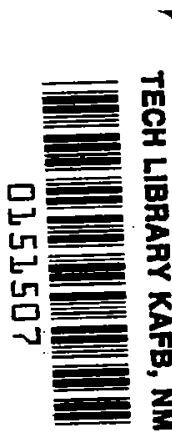

\title{
ANALYSIS OF BROKEN PLANE TRAJECTORIES FOR THE PIONEER-F MISSION
}

by Fred Teren

Lewis Research Center

Cleveland, Obio 44135

NATIONAL aERONAUtics and SPACE ADMINISTRATION - WASHINGTON, D. C. - MAY 1970 


\begin{tabular}{|c|c|c|}
\hline $\begin{array}{l}\text { 1. Report No. } \\
\text { NASA TM X-2024 }\end{array}$ & 2. Government Accession No. & 3. Rocipient's Cotalog No. \\
\hline \multirow{2}{*}{\multicolumn{2}{|c|}{$\begin{array}{l}\text { 4. Title and Subtitle } \\
\text { ANALYSIS OF BROKEN PLANE TRAJECTORIES FOR } \\
\text { THE PIONEER-F MISSION }\end{array}$}} & $\begin{array}{l}\text { 5. Report Date } \\
\text { May } 1970 \\
\end{array}$ \\
\hline & & 6. Performing Organization Code \\
\hline \multicolumn{2}{|l|}{$\begin{array}{l}\text { 7. Author(s) } \\
\text { Fred Teren }\end{array}$} & $\begin{array}{l}\text { 8. Porforming Organization Raport No. } \\
\text { E-5574 }\end{array}$ \\
\hline \multirow{3}{*}{\multicolumn{2}{|c|}{$\begin{array}{l}\text { 9. Performing Organizotion Nome and Address } \\
\text { Lewis Research Center } \\
\text { National Aeronautics and Space Administration } \\
\text { Cleveland, Ohio } 44135\end{array}$}} & $\begin{array}{l}\text { 10. Work Unit No. } \\
731-25\end{array}$ \\
\hline & & 11. Contract or Grant No. \\
\hline & & 13. Type of Report and Period Covered \\
\hline \multirow{2}{*}{\multicolumn{2}{|c|}{$\begin{array}{l}\text { 12. Sponsoring Agency Name and Address } \\
\text { National Aeronautics and Space Administration } \\
\text { Washington, D. C. } 20546\end{array}$}} & Technical Memorandum \\
\hline & & 14. Sponsoring Agency Code \\
\hline
\end{tabular}

15. Supplementary Notes

16. Abstract

A study was conducted to determine if broken plane trajectories can be used to increase the duration of the Pioneer-F launch opportunity, or, equivalently, to increase payload capability for a fixed launch opportunity duration. Optimum single conic and broken plane trajectories are calculated for the launch day - trip time combinations, and minimum trip time trajectories (for a given payload mass) are determined for each launch date. The maximum mass of spacecraft propellant required for the broken plane trajectories is calculated. Then, new minimum trip time trajectories are calculated in which the added spacecraft propellant mass is optimally utilized for all launch days. Launch opportunity duration is determined as a function of spacecraft mass for the single conic and broken plane opportunities. Values of launch vehicle injection hyperbolic velocity and declination are presented, along with values of optimum spacecraft propellant mass and correction time. The results show that the use of broken plane trajectories can provide a small increase in launch opportunity duration and/or spacecraft mass.

17. Key Words (Suggested by Author(s)) Launch opportunity Broken plane Trajectory Optimum trajectory

19. Security Classif. (of this raport) Unclassified Spacecraft

Velocity increment

18. Distribution Statement

Unclassified - unlimited

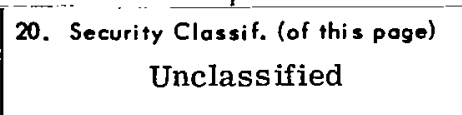
20. Security Classif. (of this page)
(s)

22. Prico* $\$ 3.00$

*For sale by the Clearinghouse for Federal Scientific and Technical Information Springfield, Virginia 22151 


\section{ANALYSIS OF BROKEN PLANE TRAJECTORIES FOR THE PIONEER - M MISSION by Fred Teren

\author{
Lewis Research Center
}

\section{SUMMARY}

A study was conducted to determine if broken plane trajectories can be used to increase the duration of the Pioneer-F launch opportunity, or, equivalently, to increase payload capability for a fixed launch opportunity duration. Optimum single conic and broken plane trajectories are calculated for the launch day - trip time combinations, and minimum trip time trajectories (for a given payload mass) are determined for each launch date. The maximum mass of spacecraft propellant required for the broken plane trajectories is calculated. Then, new minimum trip time trajectories are calculated in which the added spacecraft propellant mass is optimally utilized for all launch days. Launch opportunity duration is presented as a function of spacecraft mass for the single conic and broken plane opportunities. Values of launch vehicle injection hyperbolic velocity and declination are presented, along with the values of optimum spacecraft propellant mass and correction time. The results show that the use of broken plane trajectories can provide a small increase in launch-opportunity duration and/or spacecraft mass.

\section{INTRODUCTION}

The Pioneer-F is a Jupiter flyby mission to be launched in 1972 on an Atlas-Centaur launch vehicle with a solid-propellant third stage (TE 364-4). As has been typical for interplanetary missions, preliminary trajectory planning called for single conic trajectories to be used exclusively in establishing the launch opportunity; that is, all the required spacecraft velocity is added by the launch vehicle in the vicinity of the Earth. The heliocentric trans-Jupiter trajectory then consists of a Keplerian arc (ellipse) between Earth and Jupiter, modified only by midcourse corrections performed by the spacecraft to correct for velocity errors resulting from launch vehicle guidance system errors. 
The present study was conducted to determine if broken plane trajectories can be used to lengthen the Pioneer-F launch opportunity and/or to increase spacecraft mass capability. With broken plane trajectories, the spacecraft midcourse-correction propulsion system is used to change the trajectory velocity vector in heliocentric space. For certain launch day - arrival day combinations, usually when the heliocentric travel angle is near 180 degrees, such a maneuver can increase payload mass capability by reducing the launch vehicle velocity requirements.

Broken plane trajectories have been shown by several authors to be useful in reducing energy requirements for interplanetary missions. A recent paper by Gobetz and Doll (ref. 1) presents results for Mars orbiter missions, with primary emphasis on the 1971 and 1973 opportunities. However, reference 1 and earlier papers (refs. 2 and 3) used velocity increment, $\Delta \mathrm{V}$, as the performance index. In actual $\mathrm{m}$ ission applications, it is desired to maximize either payload capability or launch opportunity duration, subject to mission constraints. This requires consideration of spacecraft mass and propulsion system characteristics, and of launch vehicle injected mass capability as a function of magnitude and direction of the geocentric hyperbolic velocity vector.

Results are presented for spacecraft mass capability as a function of trip time for the various launch dates for single conic and broken plane trajectories. These data are crossplotted to obtain minimum trip time as a function of launch date for selected values of spacecraft mass. The number of possible launch days is then determined as a function of spacecraft mass for different values of maximum allowable trip time. In addition, the values of hyperbolic velocity, declination of the geocentric asymptote, optimum spacecraft propellant mass, and time of spacecraft propulsive maneuver are also presented.

\section{METHOD OF ANALYSIS}

The mission strategy assumed in this study is to select, for each launch date, the shortest trip time consistent with launch vehicle capability and spacecraft mass. Launch vehicle injected mass capability is calculated as a function of hyperbolic excess velocity and the declination of the geocentric asymptote. The spacecraft propellant mass required for the plane change is calculated by using the specific impulse of the spacecraft midcourse correction propulsion system, which was initially designed to correct for guidance system errors. To use this spacecraft propulsion system to effect plane changes, additional propellants, propellant tank capacity, and possible structural modifications would be required for the spacecraft. The existing spacecraft propulsion system uses monopropellant hydrazine, with a specific impulse $I_{s p}$ of 225 seconds. 
This value of $I_{s p}$ is used in this report. However, the increase in propellant tank mass and, possibly in structural mass, is not evaluated in this report.

\section{Calculation of Launch Opportunity}

The launch opportunity is determined by calculating, for each launch day - trip time combination, the maximum spacecraft mass which can be delivered, both for single conic (no spacecraft propulsive maneuver used) and broken plane trajectories. Then, for each launch day, the minimum trip time is determined (for a given spacecraft mass). This trip may be either a single conic or a broken plane transfer. The required spacecraft propellant loading is the maximum required on any day in the launch opportunity. If it is assumed that this spacecraft propellant must be tanked for all days in the opportunity (including those days when single conic trajectories are optimum), a new set of optimum transfers must be determined, in which the full amount of added spacecraft propellant must be consumed in an optimum manner on each launch day. The resulting minimum trip times for the modified opportunity are longer than the previously calculated values, since it was not optimum to use the full amount of propellant except on one launch day.

The Fletcher-Powell gradient method (ref. 4) is used to determine the mass of spacecraft propellant, and the time and direction of spacecraft burn, which maximize payload capability for each launch day - trip time combination.

\section{Calculation of Trajectories}

The single conic launch opportunity is determined by calculating the spacecraft mass capability for each launch day - trip time combination. The required trajectories are calculated by solving Lambert's problem (ref. 5). Then, the required $\Delta \mathrm{V}$ at Earth is rotated from heliocentric to geocentric coordinates, and the required hyperbolic velocity $\mathrm{V}_{\mathrm{H}}$ and declination $\delta$ and right ascension $\varphi$ of the outgoing asymptote are determined. Launch vehicle injected mass capability is calculated as a function of $V_{H}$ and $\delta$ by using an empirical formula obtained from integrated launch vehicle trajectories. The value of $\varphi$ simply determines the optimum launch time, corresponding to a zeroduration launch window. However, since a finite launch window is required on each launch day, the injected weight must be reduced below its value for the optimum launch time and $\varphi$. The reduction is not considered herein, but unpublished results have shown the mass loss to be from 5 to 10 pounds mass (2.3 to $4.6 \mathrm{~kg}$ ) for a 30 -minute launch window. 
The optimality of the single conic trajectories is checked by calculating the magnitude of the variational primer vector along the reference trajectory. This procedure is described in appendix B. (All symbols are defined in appendix A.) If the single-conic trajectory is found to be nonoptimum, an optimum broken plane trajectory is calculated for the same launch day - trip time combination. The optimum broken plane trajectory is determined by using the Fletcher-Powell gradient method (ref. 4) to optimize the time, magnitude, and direction of the spacecraft impulse.

Once the optimum trajectories are determined, the launch opportunity is defined by selecting, for each launch day, the shortest trip time which allows the required spacecraft mass to be achieved. The resulting trips may be either single conic or broken plane transfers.

\section{RESULTS AND DISCUSSION}

The spacecraft mass capability is shown as a function of trip time for three launch days in figure 1. Net spacecraft mass is defined as the injected spacecraft mass less

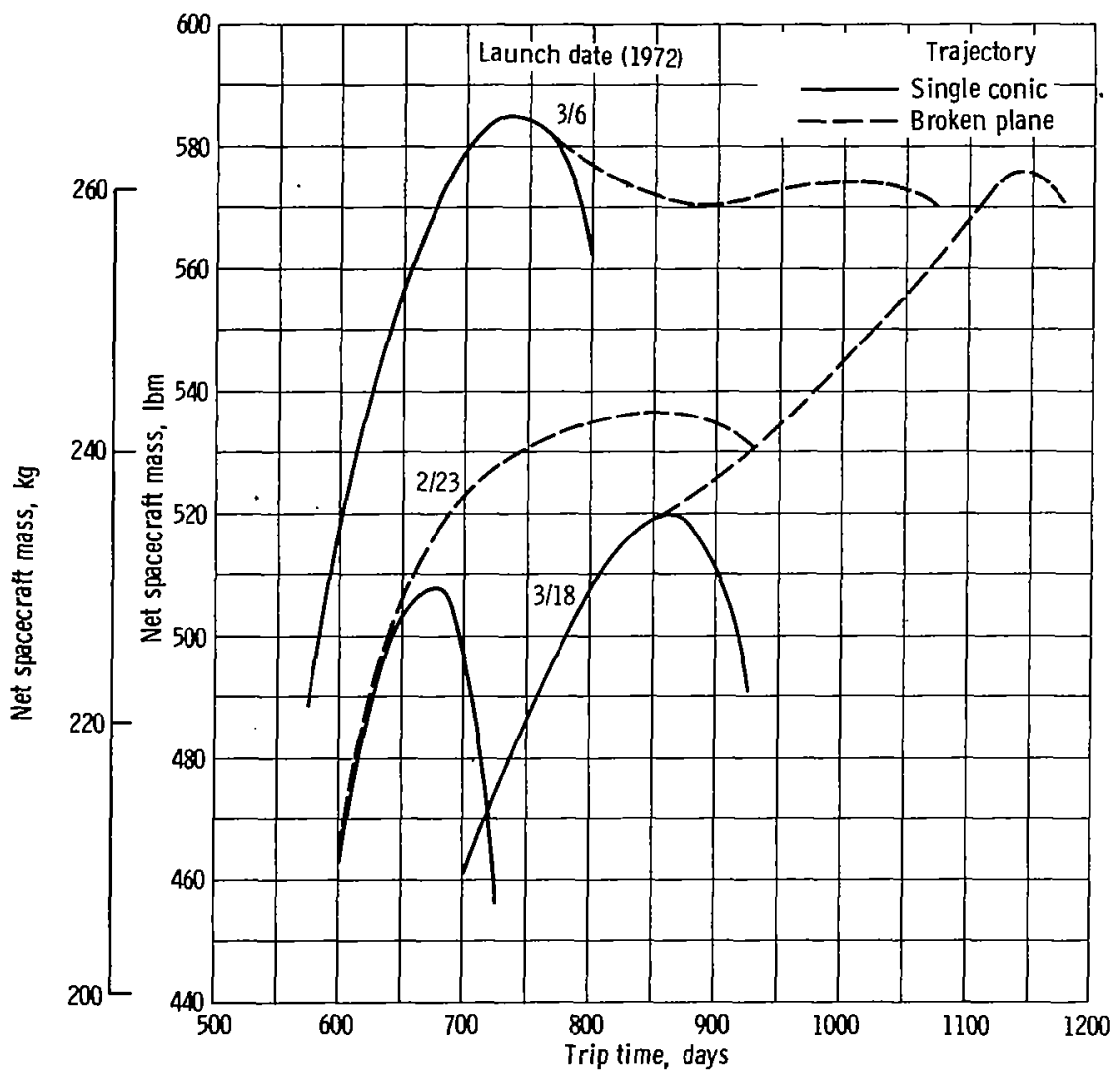

Figure 1. - Maximum net spacecraft mass as a function of trip time for three launch days. Single conic and broken plane trajectories. 
the amount of spacecraft propellant added for the spacecraft impulse (for the broken plane trajectories). For launch on February 23, 1972, which is early in the launch opportunity (as will be shown later), broken plane trajectories are optimum for all trip times and all values of spacecraft mass. However, the difference between single conic and broken plane capability is small for trip times up to 650 days. For a spacecraft mass of 500 pounds mass $(227 \mathrm{~kg})$, single conic trajectories could be used with a very slight increase in trip time. However, for a spacecraft mass of 510 pounds mass $(232 \mathrm{~kg})$ or greater, single conic trajectories cannot be used. Broken plane trajectories, on the other hand, can be used for values of spacecraft mass up to 535 pounds mass $(243 \mathrm{~kg})$ with trip times of 850 days or less.

For launch on March 6, 1972 (the center of the opportunity), single-conic trajectories are optimum for all values of spacecraft mass, assuming that the shortest trip time is desired. The single conic trajectories are also optimum for a March 18, 1972, launch date (near the end of the single conic opportunity) if the spacecraft mass is 520 pounds mass $(236 \mathrm{~kg})$ or less. If the spacecraft mass is between 520 and 575 pounds mass (236 and $261 \mathrm{~kg}$ ), broken plane trajectories may be used. The required trip times, however, are rather long, ranging from 850 days for a 520 -pound-mass $(236-\mathrm{kg})$ spacecraft to more than 1100 days for a 570 -pound-mass $(259-\mathrm{kg})$ spacecraft.

Similar data are displayed in figure 2 for a family of launch dates. Figure 2 differs

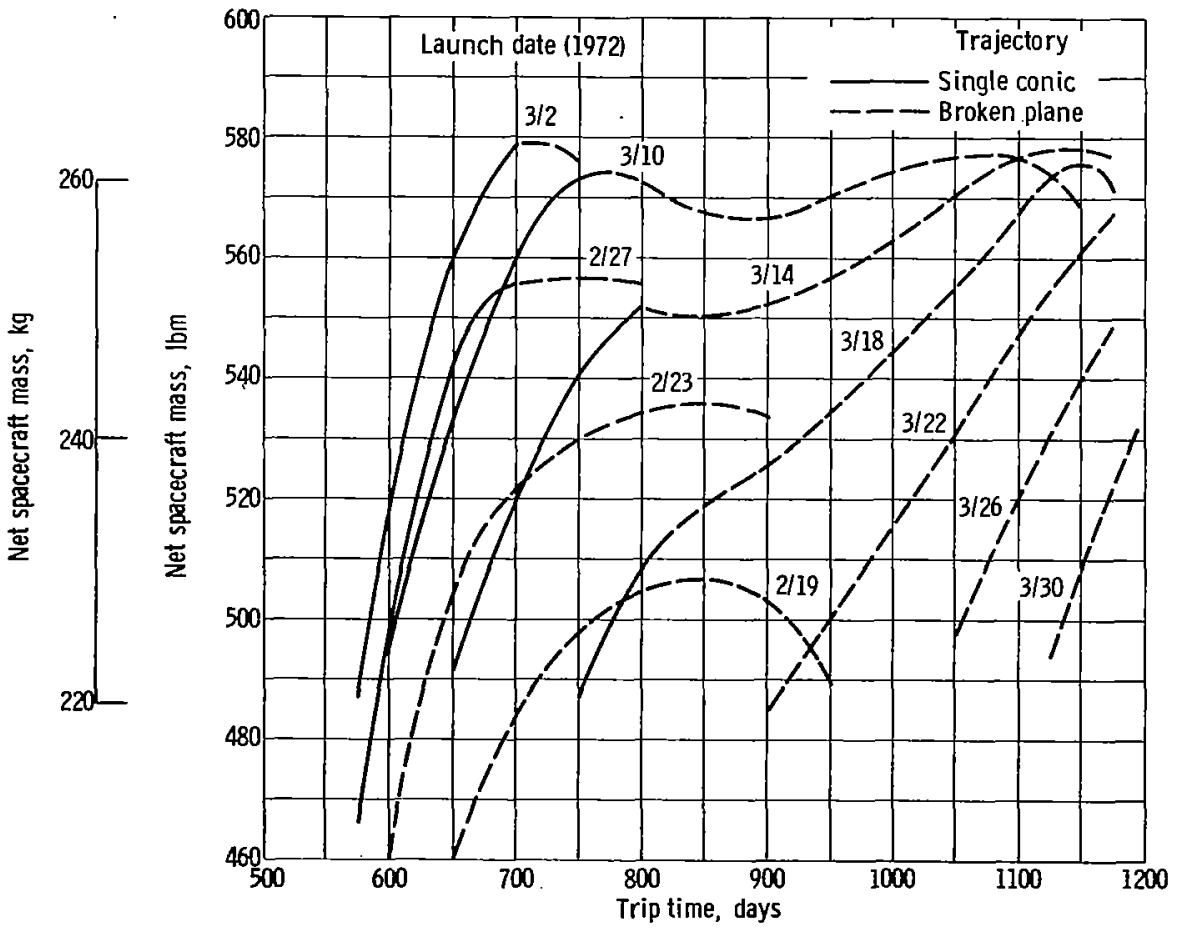

Figure 2. - Maximum net spacecraft mass as a function of trip time for a family of launch dates. Single conic and broken plane trajectories (only optimum shown). 
from figure 1 in that only the optimum trajectory (single conic or broken plane) is shown in figure 2 for each launch day and trip time. The following trends are readily observable from figure 2: For early launch dates, broken plane trajectories are optimum. As the launch date increases to about February 27, 1972, single conic trajectories become optimum and remain so up to about March 18. At this time, broken plane trajectories become optimum again.

The data in figure 2, and other data of the same type for other'launch days, are crossplotted to determine the launch opportunity. For each launch day, the minimum trip time is determined for a given spacecraft mass, and plotted as a function of launch date in figure 3 . The required trip times increase with spacecraft mass. For spacecraft masses of 540 pounds mass $(245 \mathrm{~kg})$ or less, broken plane trajectories supply additional launch days prior to the opening and after the close of the single conic opportunity. The required broken plane trip times for launch in late February 1972, are in the same range as those required for single conic trajectories. However, for broken plane trajectories in late March, the trip times increase rapidly. The curves were arbitrarily terminated at a trip time of 1200 days, although they could have been con-

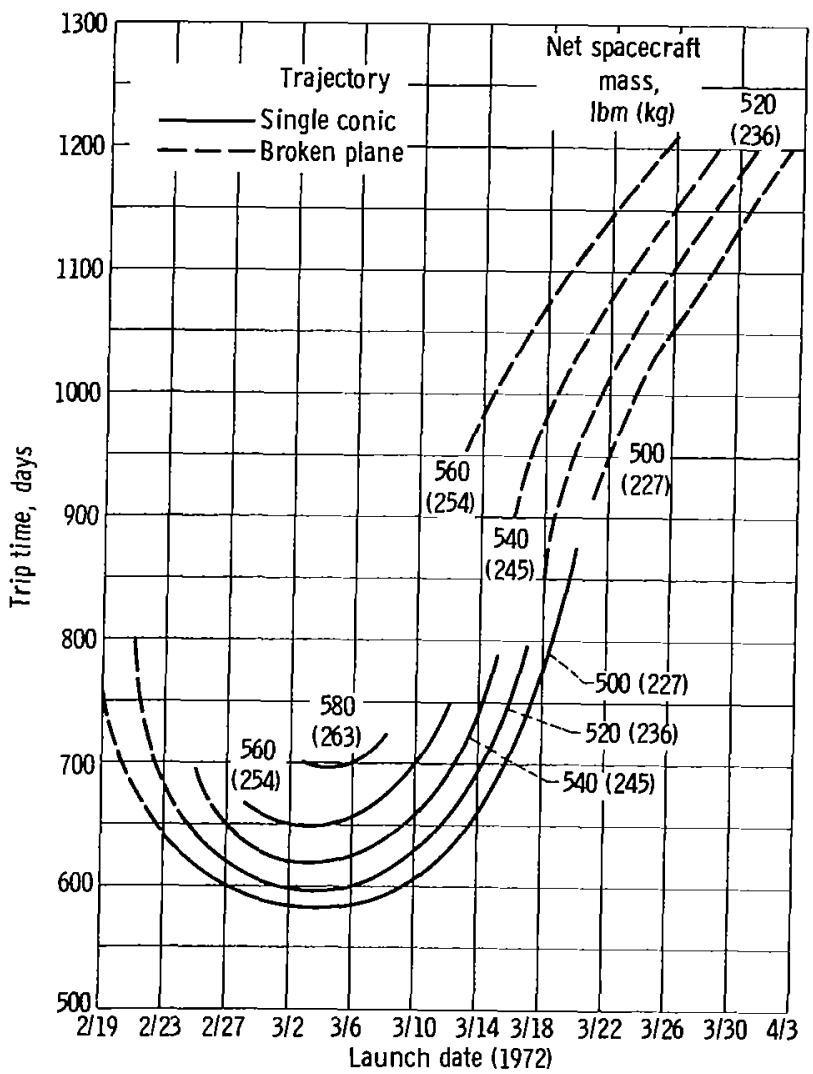

Figure 3. - Minimum trip time as a function of launch date. Optimum spacecraft propellant mass. 
tinued to give additional launch dates with trip times longer than 1200 days. For spacecraft mass greater than 540 pounds mass $(245 \mathrm{~kg})$, broken plane trajectories do not exist prior to the opening of the single conic opportunity.

From figure 3, the number of available launch days may be calculated for each value of spacecraft mass. For a spacecraft mass of 520 pounds mass $(227 \mathrm{~kg})$, a 23-day launch opportunity is available with single conic trajectories. Maximum trip times of 725 to 875 days are required. The launch opportunity can be increased to 27 days by using broken plane trajectories with no increase in maximum trip time, or to 39 days if $\mathbf{1 2 0 0}$ day trips are permitted. If it is decided that a 23-day launch opportunity is adequate, then broken plane trajectories allow an increase in spacecraft mass to 540 pounds mass $(245 \mathrm{~kg}$ ) for a 975 -day maximum trip time, or to 560 pounds mass $(254 \mathrm{~kg})$ if 1125-day trips are allowed. Thus, it is seen that the broken plane trajec- . tories can be used to increase the number of available launch days, or the spacecraft mass for a fixed number of launch days, or a combination of both.

The mass of spacecraft propellant required for the broken plane transfers is presented in figure 4 as a function of launch date, for various values of spacecraft mass.

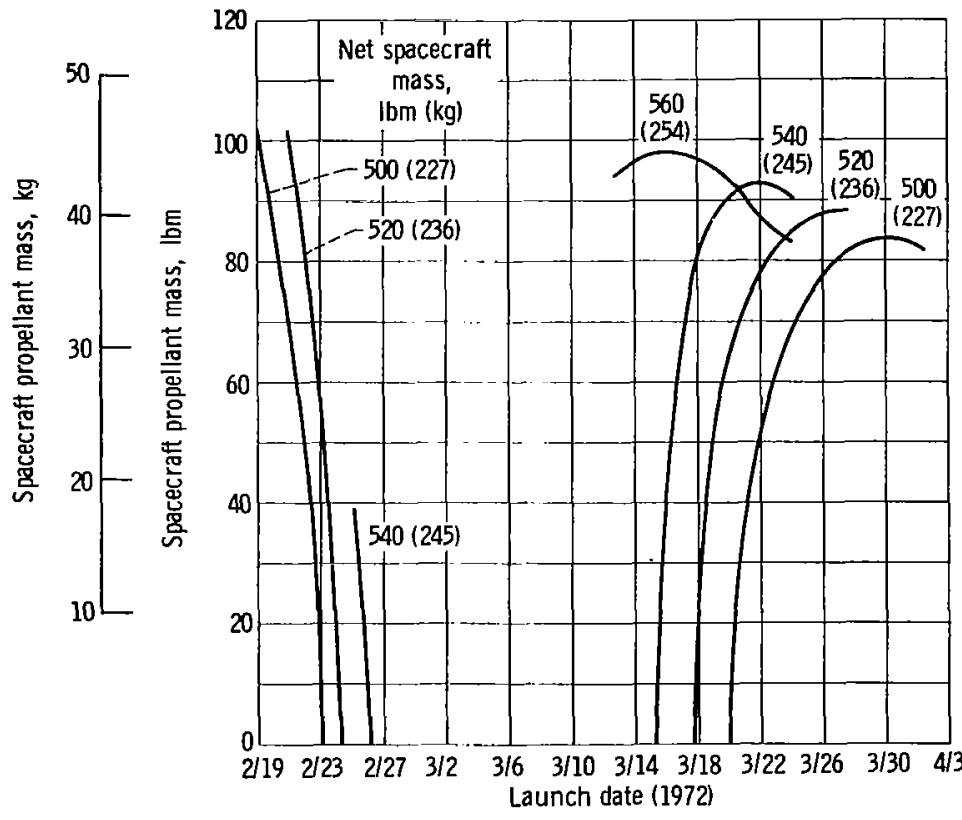

Figure 4. - Optimum spacecraft propeliant mass as a function of launch date for broken plane trajectories. 


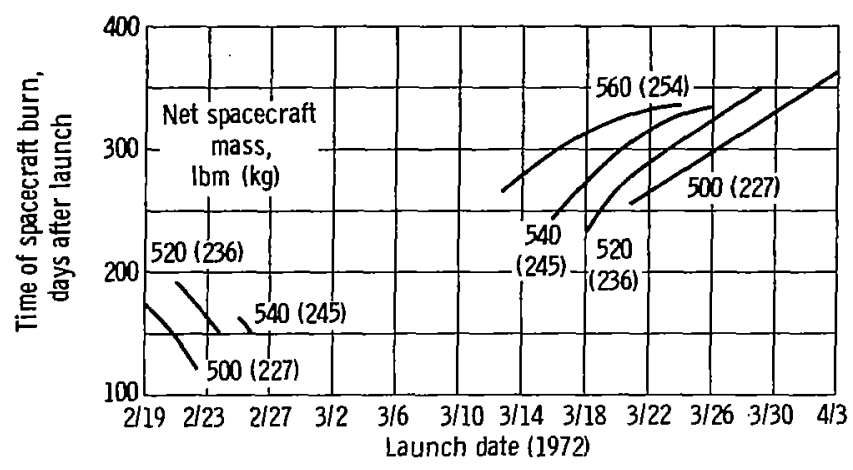

Figure 5. - Optimum time of spacecraft correction as a function of launch date for broken plane trajectories.

The corresponding time of the spacecraft propulsive maneuver is presented in figure 5. These values correspond to the minimum trip times for each launch date and spacecraft mass, as presented in figure 3. It can be seen from figure 4 that about 100 pounds mass $(45 \mathrm{~kg})$ of spacecraft propellant is required to take full advantage of the broken plane trajectories. Increased propellant tank capacity (hence, mass) would be required for this additional propellant mass. In addition, some structural modifications might be required for the spacecraft. These mass increases are not considered in this report. Figure 5 shows that the optimum spacecraft burn times occur about 100 to 200 days after launch for broken plane transfers early in the opportunity, while the corresponding times late in the opportunity range from 250 to 350 days after launch.

The required values of launch vehicle hyperbolic velocity and declination of the geocentric asymptote are presented in figures 6 and 7 , respectively. For single conic transfers, the hyperbolic velocity is very nearly constant for a given value of spacecraft mass. The required launch-vehicle hyperbolic velocity decreases when broken plane transfers are used, because the spacecraft supplies some of the required velocity. The values of declination shown in figure 7 become less negative when broken plane transfers are used. This is reflected in less southerly launch azimuths.

From the results presented in figure 4 , about 100 pounds mass (45 kg) of spacecraft propellant mass should be added to take full advantage of the broken plane trajectories. A question that arises is how this propellant should be utilized in the single conic opportunity and on those days when broken plane trajectories do not require the full 100 pounds mass $(45 \mathrm{~kg})$. The optimum procedure would be simply to load the optimum amount of spacecraft propellant (if any) each launch day. If this procedure is followed, the launch opportunity will be unaffected, except for a slight increase in spacecraft system mass resulting from increased tankage and structural modifications. However, it is possible that spacecraft propellants may not be varied throughout the launch opportunity. In this 


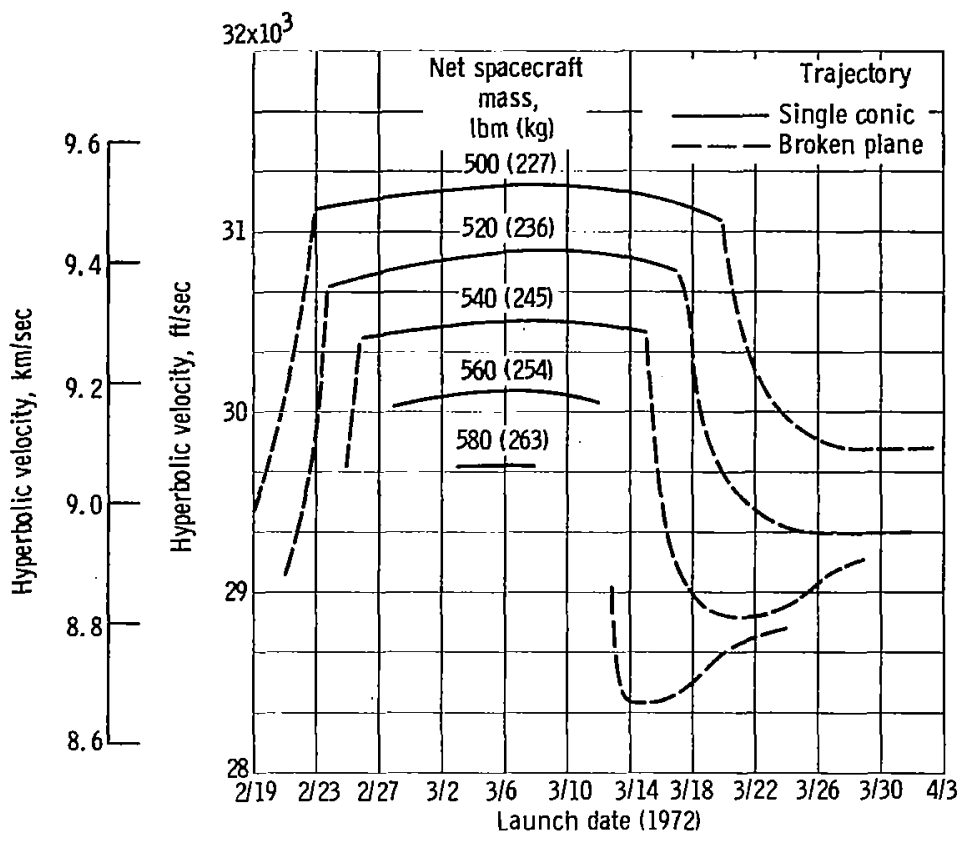

Figure 6. - Injection hyperbolic velocity as a function of launch date. Optimum spacecraft propellant mass; single conic and broken plane trajectories.

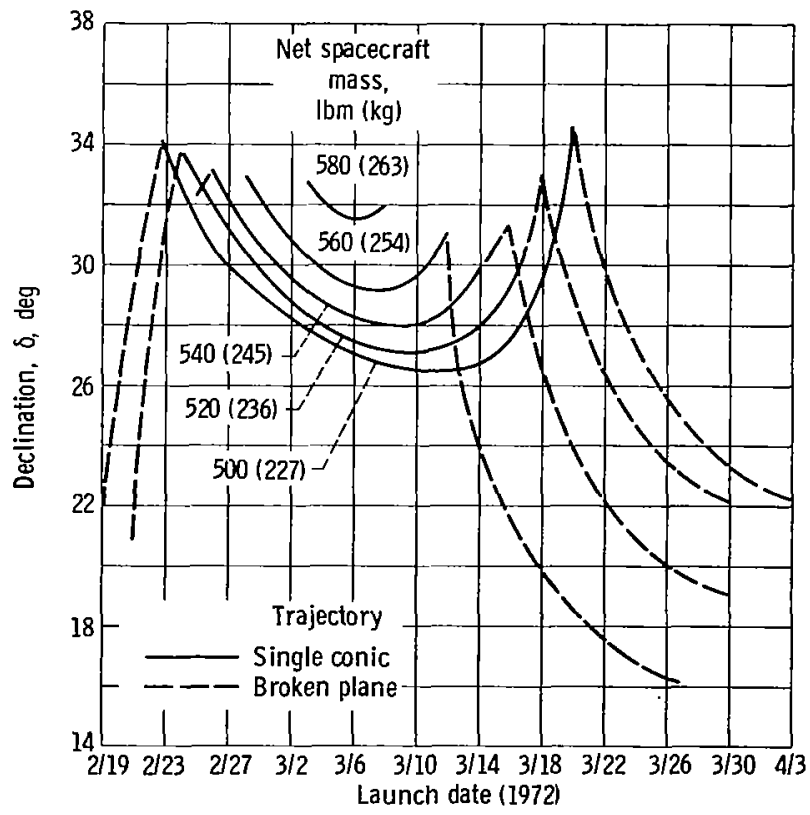

Figure 7. - Declination of the geometric asymptote as a function of launch date. Optimum spacecraft propellant mass; single conic and broken plane trajectories. 
case, a fixed spacecraft propellant load must be tanked and utilized in an optimum manner.

If the spacecraft is forced to contain propellants in excess of the optimum amount, the spacecraft mass capability will decrease for each launch day - trip time combination. The trip times shown in figure 3 cannot then be used. A new set of optimum trajectories must be obtained for the launch day - trip time combinations, in which 100 pounds mass $(45 \mathrm{~kg})$ of spacecraft propellant must be optimally utilized for each case.

Spacecraft mass capability is presented as a function of trip time for several launch days in figure 8 for the restricted optimum trajectories. Comparison with figures 1

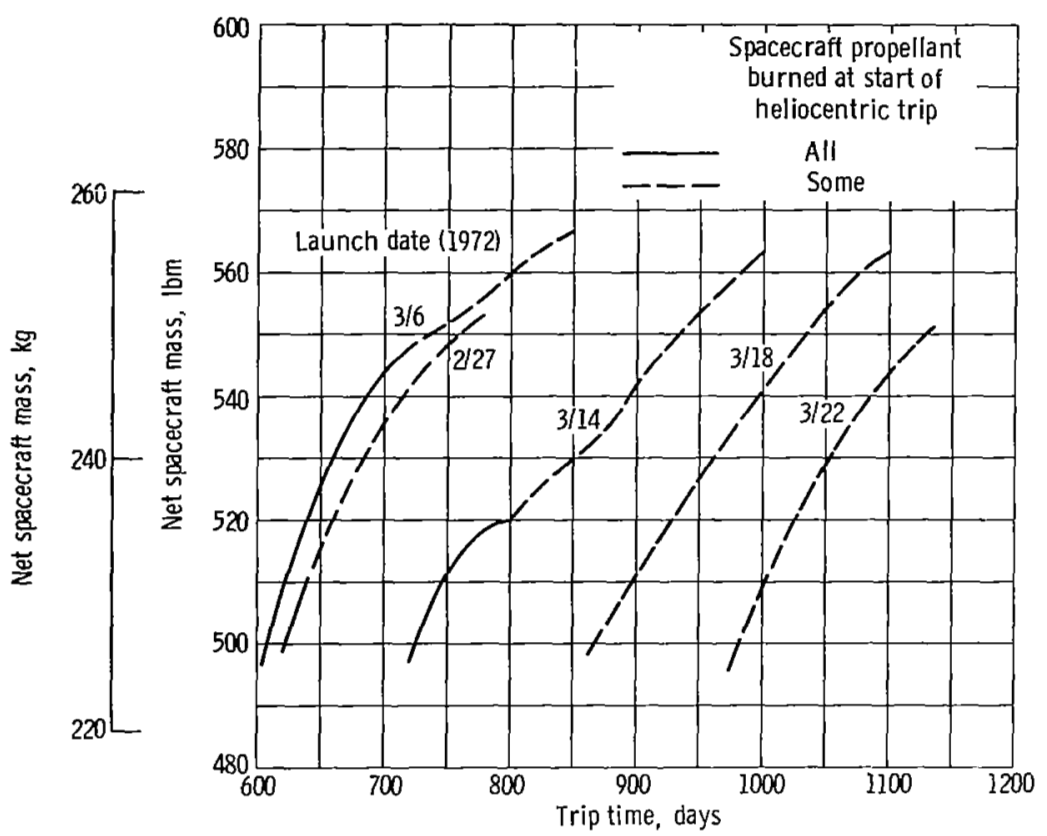

Figure 8. - Maximum net spacecraft mass as a function of trip time for several launch days. Spacecraft propellant mass, 100 pounds mass $(45 \mathrm{~kg})$.

and 2 shows that the spacecraft mass capability has decreased for all launch day - trip time combinations. On launch days which had previously been single conic days (such as March 6), it is optimum to use the propellant as soon as possible after spacecraft separation (from the launch vehicle) for values of spacecraft mass to about 550 pounds mass $(250 \mathrm{~kg})$. It is assumed that about 1 day is required to obtain preliminary tracking information and proper spacecraft orientation. For the values of hyperbolic velocity required for this mission, the spacecraft has essentially escaped from the Earth's sphere of influence after 1 day, so that the spacecraft burn takes place in heliocentric space. However, the spacecraft is very near to Earth, relative to heliocentric dimensions. 
On launch days which previously had required broken plane trajectories, part of the spacecraft propellant is burned near the Earth; with the remainder (at least as much as used in the unrestricted case) used to construct essentially the same broken plane trajectory as previously. For a given launch date and trip time, the payload degradation resulting from the 100 pounds mass $(45 \mathrm{~kg}$ ) of spacecraft propellant is smaller when the unrestricted trip had been a broken plane transfer. This result is expected, since the additional spacecraft propellant mass is a smaller amount above the optimum for these cases.

The minimum trip times for the restricted-spacecraft-propellant-mass case are plotted in figure 9 as a function of launch date and are illustrated for several values of

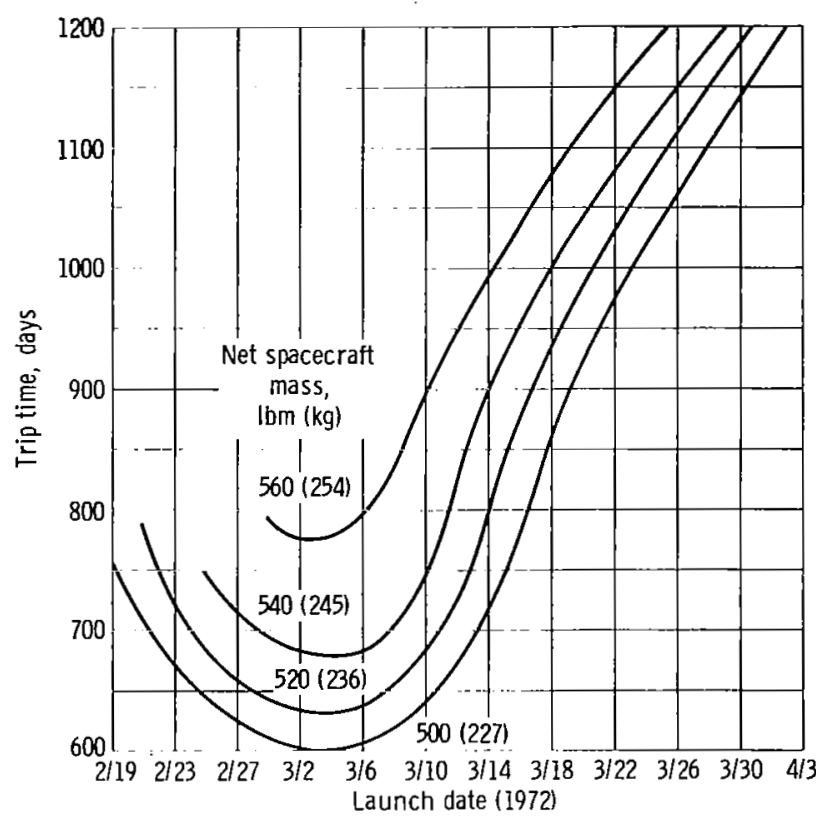

Figure 9. - Minimum trip time as a function of launch date. Spacecraft propellant mass, 100 pounds mass $(45 \mathrm{~kg})$.

spacecraft mass, as in figure 3. The increase in minimum trip time can be seen by comparison with figure 3 . Spacecraft mass of 580 pounds mass $(263 \mathrm{~kg})$ or greater cannot be delivered when 100 pounds mass (45 kg) of spacecraft propellant must be carried.

The duration of launch opportunity for the restricted case may be determined from figure 9 and may be compared to the results from figure 3 . The number of additional broken plane days is decreased by at most 1 day if at all, for all values of maximum trip time and spacecraft mass.

The mass of spececraft propellant utilized in a broken plane manner is shown in figure 10 for the launch day - trip time combinations defined in figure 9. The trajectories launched in early to middle March 1972, are single conic type for a spacecraft mass less than 560 pounds mass $(254 \mathrm{~kg})$, in that all the propellant is burned near 


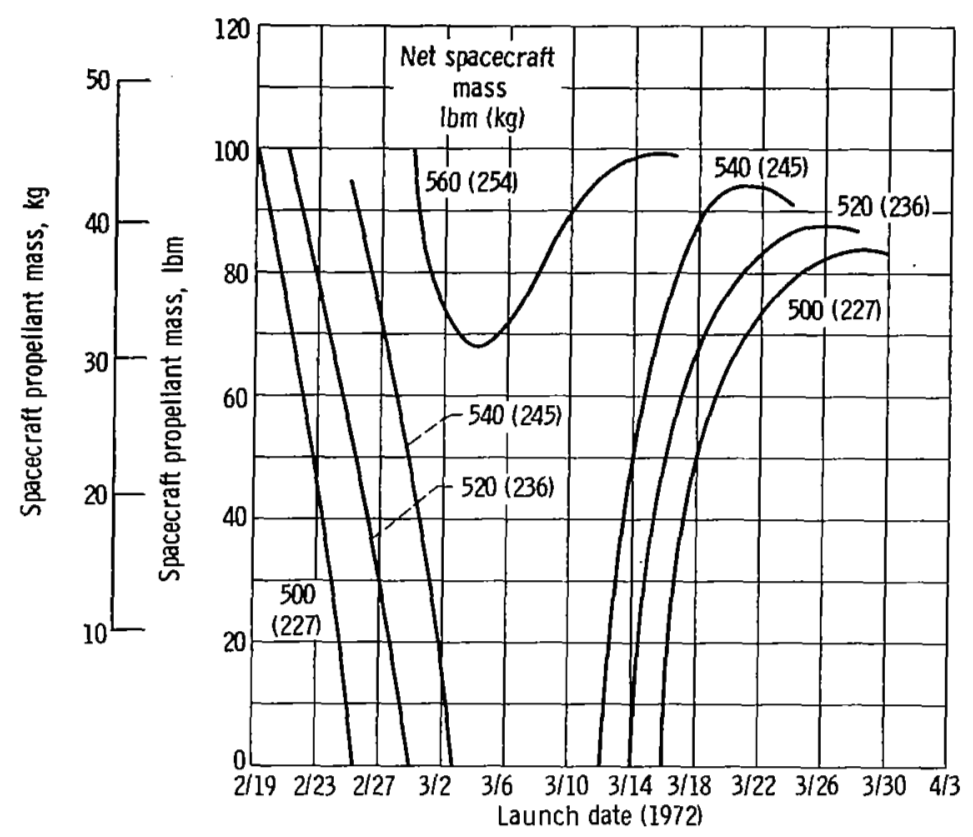

Figure 10. - Mass of broken-plane-type spacecraft propellant as a function of launch date. Total spacecraft propellant mass $(45 \mathrm{~kg})$.

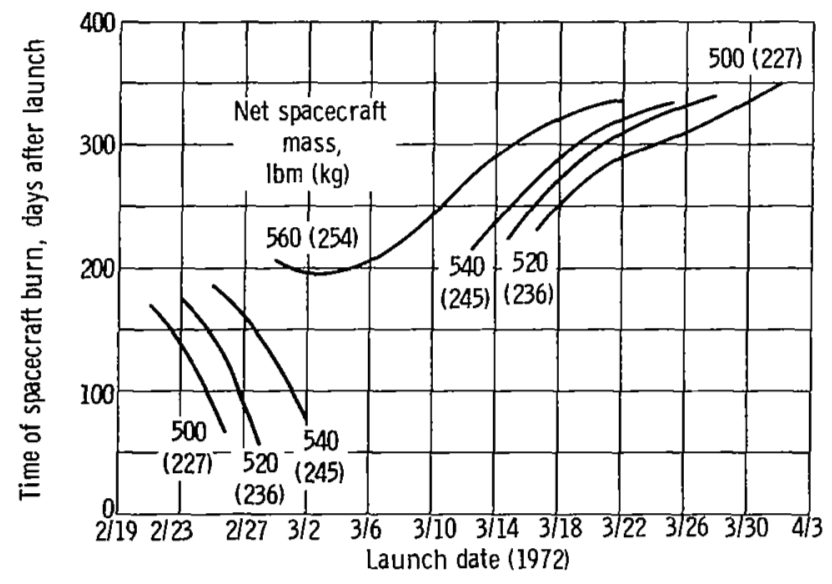

Figure 11. - Optimum time of spacecraft correction as a function of launch date. Spacecraft propellant mass, 100 pounds mass ( $45 \mathrm{~kg}$ ). 
Earth. For a spacecraft mass of 560 pounds mass $(254 \mathrm{~kg}$ ) or greater, all the trajectories are broken plane type. The mass of broken plane type spacecraft propellant shown in figure 10 is always at least as large as the corresponding optimum value presented in figure 4. As in the unrestricted case, the broken plane type propellant mass increases to nearly 100 pounds mass $(45 \mathrm{~kg})$ at both ends of the launch opportunity.

The optimum spacecraft correction times corresponding to the launch day - trip time combinations in figure 9 and the propellant mass in figure 10 are shown in figure 11. These values do not differ appreciably from those presented in figure 5.

\section{CONCLUDING REMARKS}

The results presented show that broken plane transfers can be used to increase the duration of the Pioneer-F launch opportunity. Broken plane trajectories which deliver the required spacecraft mass are available both before the opening and after the close of the single conic launch opportunity. The required trip times before the single conic opening are in the same range as those required for the single conic trips. However, the required trip times after the closing of the single conic opportunity increase rapidly with launch date. Therefore, the possible increase in available launch days depends on the maximum allowable trip time.

To take full advantage of the broken plane trajectories, the existing spacecraft propellant tanks must be resized to allow up to 100 additional pounds mass (45 $\mathrm{kg}$ ) of propellant. For best performance, the amount of spacecraft propellant loaded must be varied from day to day. If this is not possible, the additional 100 pounds mass (45 kg) is loaded and utilized on each launch day. The required trip times for this case are somewhat longer than if the propellant can be optimally tanked, but no more than 1 potential launch day is lost for any value of spacecraft mass.

The addition of spacecraft propellant will cause an increase in propellant tank mass, and possibly in structural mass. This mass increase has not been evaluated or considered in this report. In addition, it may be possible in certain cases to combine a required midcourse correction with a planned spacecraft burn, thus reducing the total amount of propellant required for the two maneuvers. This may offset some, or all, of the additional propellant tank and structure mass.

The results presented show that the use of broken plane trajectories gives only a small improvement for the Pioneer-F mission. It should be recognized, however, that these results are only valid for the 1972 Jupiter flyby opportunity. The advantage of 
broken plane trajectories depends on the existing geometry, which varies from opportunity to opportunity. However, the report serves to illustrate the tradeoffs which must be considered in evaluating broken plane trajectories.

\section{Lewis Research Center,}

National Aeronautics and Space Administration, Cleveland, Ohio, March 17, 1970,

731-25. 


\section{APPENDIX A}

\section{SYMBOLS}

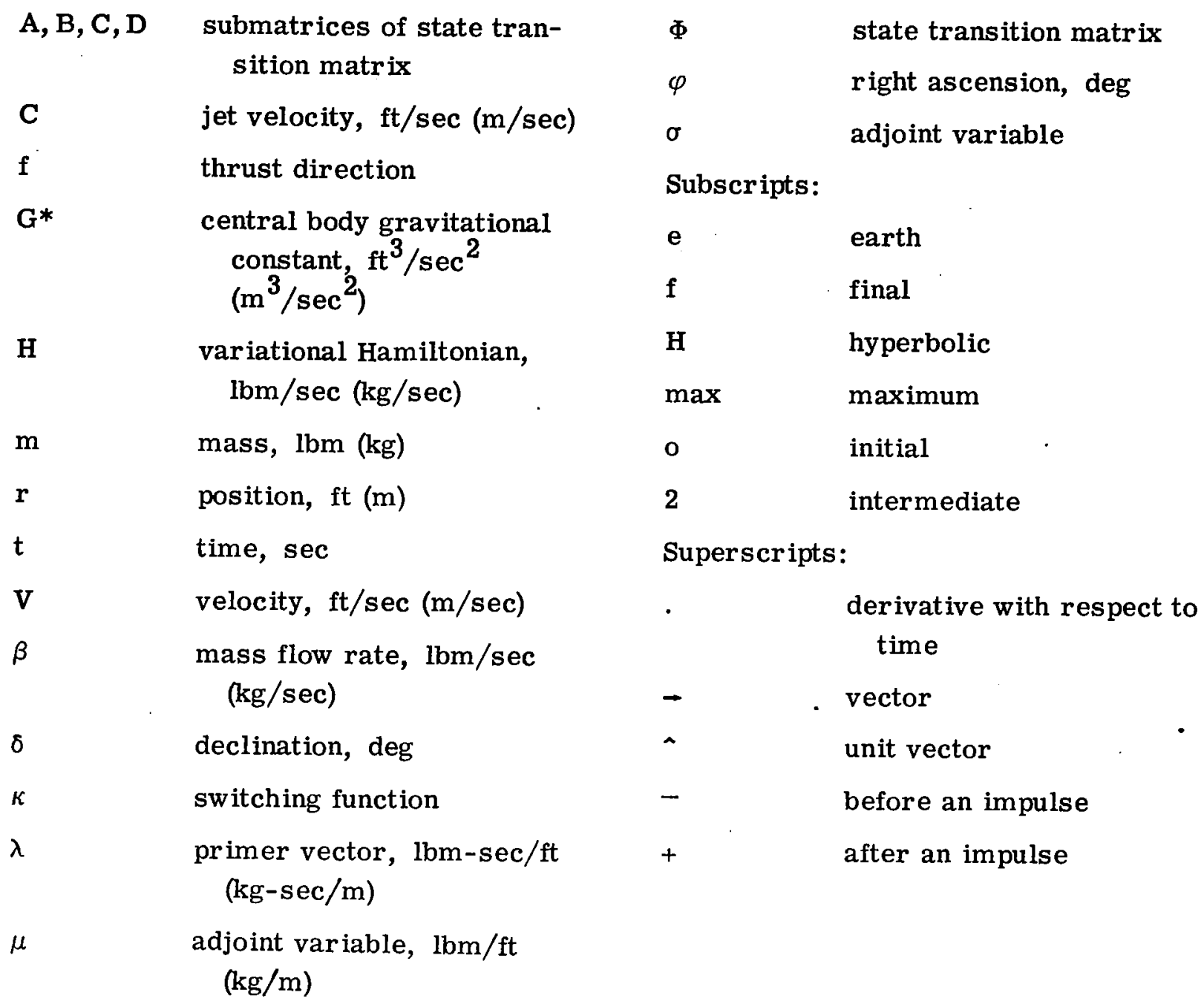




\section{APPENDIX B}

\section{MATHEMATICAL ANALYSIS}

The mathematical problem to be solved is to find optimal fixed-transfer-time impulsive transfers between two fixed positions in three-dimensional space with an inverse-square force field acting. This problem was studied by Pines (ref. 6), and the important results are repeated here for convenience.

The equations of motion are

$$
\begin{gathered}
\dot{\overrightarrow{\mathrm{V}}}=-\frac{\mathrm{G}^{*}}{\mathrm{r}^{3}} \overrightarrow{\mathrm{r}}+\frac{\mathrm{C} \beta}{\mathrm{m}} \hat{\mathrm{f}} \\
\dot{\overrightarrow{\mathrm{r}}}=\overrightarrow{\mathrm{v}} \\
\dot{\mathrm{m}}=-\beta
\end{gathered}
$$

where the controls are the thrust direction $\hat{\mathrm{f}}$ and the flow rate $\beta$, which are restricted by $|\hat{\mathrm{f}}|=1$ and $\beta \geq 0$. The variational Hamiltonian is

$$
\mathbf{H}=-\frac{\mathrm{G}^{*}}{\mathbf{r}^{3}} \bar{\lambda} \cdot \overrightarrow{\mathbf{r}}+\frac{\mathrm{C} \beta}{\mathrm{m}} \vec{\lambda} \cdot \hat{\mathbf{f}}+\vec{\mu} \cdot \overrightarrow{\mathrm{V}}-\sigma \beta
$$

and the adjoint equations are

$$
\begin{gathered}
\dot{\vec{\lambda}}=-\vec{\mu} \\
\dot{\vec{\mu}}=\frac{\mathrm{G}^{*}}{\mathrm{r}^{3}} \vec{\lambda}-3 \frac{\mathrm{G}^{*}}{\mathrm{r}}(\vec{\lambda} \cdot \overrightarrow{\mathrm{r}}) \overrightarrow{\mathrm{r}} \\
\dot{\sigma}=\frac{\mathrm{C} \beta}{\mathrm{m}^{2}} \vec{\lambda} \cdot \hat{\mathrm{f}}
\end{gathered}
$$

To maximize $H$ with respect to $\hat{f}$ and $\beta$,

$$
\hat{\mathbf{f}}=\hat{\lambda}
$$


and

$$
\begin{gathered}
\beta=0 \quad \text { if } \kappa=\frac{\mathrm{C}}{\mathrm{m}} \boldsymbol{\lambda} \cdot \hat{\mathbf{f}}-\sigma=\frac{\mathrm{C}}{\mathrm{m}} \lambda-\sigma<0 \\
\beta \rightarrow \infty \quad \text { if } \kappa>0
\end{gathered}
$$

and $\beta$ is indeterminate if $\kappa=0$. The singular arc, $\kappa=0$, was treated by Pines but will not be considered here. Pines showed that impulses occur when $\kappa=0$, and furthermore, that $\kappa \leq 0$ for the entire (optimal) trajectory. In addition, he showed that the value of $\mathrm{m} \sigma \cdot$ is continuous throughout the trajectory.

For the present problem, the impulses are supplied by the spacecraft with jet velocity $C$. The launch vehicle is treated by allowing variations in the initial mass and velocity in the transversality equation. Consider first the problem of transferring between fixed positions $\vec{r}_{o}$ and $\vec{r}_{f}$ with a fixed transfer time, and let all of the required velocity at $\vec{r}_{\mathrm{o}}$ be supplied by the launch vehicle. No impulse is required at $\vec{r}_{f}$ since the mission is a flyby with no specification on final velocity. The required $\Delta \vec{V}_{0}$ is obtained by solving Lambert's problem. The transversality equation for this problem is

$$
(\vec{\lambda} \cdot d \vec{V}+\vec{\mu} \cdot d \vec{r}+\sigma d m-H d t)_{t_{f}}-(\vec{\lambda} \cdot d \vec{V}+\vec{\mu} \cdot d \vec{r}+\sigma d m-H d t)_{t_{0}}-d m_{f}=0
$$

where $m_{f}$ is the quantity to be maximized. The initial and final positions and transfer time are fixed so that

$$
\mathrm{d} \overrightarrow{\mathbf{r}}_{\mathrm{f}}=\mathrm{d} \overrightarrow{\mathbf{r}}_{\mathrm{o}}=\overrightarrow{0}
$$

and

$$
\mathrm{H}_{\mathrm{f}}=\mathrm{H}_{\mathrm{O}}
$$

The final velocity is free, so that $\vec{\lambda}_{f}=\overrightarrow{0}$. Also, $m_{0}$ (the injected spacecraft mass) is a function of $\Delta \vec{V}=\vec{V}_{o}-\vec{V}_{e}$, where $\vec{v}_{e}$ is the Earth's heliocentric velocity. Then $\mathrm{dm}_{0}$ can be expressed as

$$
d m_{0}=\vec{\nabla} \vec{V}_{o} m_{o} \cdot d \vec{V}_{0}
$$

and since $\vec{V}_{0}$ is free, 


$$
\vec{\lambda}_{0}=-\sigma_{0} \vec{\nabla}_{v_{0}} m_{0}
$$

Also, $m_{\mathrm{f}}$ is free, so that $\sigma_{\mathrm{f}}=1$; and since $m_{\mathrm{o}}=\mathrm{m}_{\mathrm{f}}$ (there is no spacecraft impulse), it follows that $\sigma_{0}=1$. Then

$$
\vec{\lambda}_{\mathrm{o}}=-\vec{\nabla}_{\mathrm{v}_{\mathrm{o}}} \mathrm{m}_{\mathrm{o}}
$$

From the known values of $\vec{\lambda}_{f}$ and $\vec{\lambda}_{\mathrm{o}}$, the values of $\vec{\lambda}$ and $\vec{\mu}$ can be calculated at any point on the trajectory by using the state transition matrix as discussed by Pines (ref. 6) and presented explicitly by Danby (ref. 7). If the state transition matrix is partitioned,

$$
\Phi=\left(\begin{array}{ll}
A & B \\
C & D
\end{array}\right)
$$

then $\vec{\lambda}_{\mathrm{f}}=\mathrm{A} \vec{\lambda}_{\mathrm{o}}+\mathrm{B} \vec{\mu}_{\mathrm{o}}=\overrightarrow{0}$ and $\vec{\mu}_{\mathrm{o}}=-\mathrm{B}^{-1} \mathrm{~A} \vec{\lambda}_{\mathrm{o}}$. The values of $\vec{\lambda}$ and $\vec{\mu}$ at any point along the trajectory are then obtained by application of the state transition matrix to $\vec{\lambda}_{0}$ and $\vec{\mu}_{0}$.

To test the optimality of this single conic trajectory, the maximum value of $\vec{\lambda}$ along the trajectory is determined. If $\mathbf{C} \lambda_{\max } / \mathrm{m}<1$, then no spacecraft impulse is desired along the trajectory, and the single conic trajectory is an optimum (probably, but not necessarily, a global optimum). If $C \lambda_{\max } / \mathrm{m}>1$, then the single conic trajectory is not locally optimum. The time and position vector $t_{2}$ and $\vec{r}_{2}$, corresponding to $\lambda_{\max }$ are then calculated, modified slightly, and used as first estimates of the optimum time and position of the spacecraft impulse. Then, trajectories are constructed between $\vec{r}_{o}$ and $\vec{r}_{2}$ and between $\vec{r}_{2}$ and $\vec{r}_{f}$, with trip times $t_{2}-t_{0}$ and $t_{f}-t_{2}$, respectively. The primer vector along this trajectory is

$$
\begin{aligned}
& \vec{\lambda}_{\mathrm{o}}=-\frac{\mathrm{m}_{\mathrm{f}}}{\mathrm{m}_{\mathrm{o}}} \vec{\nabla}_{\overrightarrow{\mathrm{v}}_{\mathrm{o}}} \mathrm{m}_{\mathrm{o}} \\
& \vec{\lambda}_{2}=\frac{\left(\overrightarrow{\mathrm{v}}_{2}^{+}-\overrightarrow{\mathrm{v}}_{2}^{-}\right)}{\left|\overrightarrow{\mathrm{v}}_{2}^{+}-\overrightarrow{\mathrm{v}}_{2}^{-}\right|} \frac{\mathrm{m}_{\mathrm{f}}}{\mathrm{C}}
\end{aligned}
$$

The values of $\vec{\mu}_{2}^{-}$and $\vec{\mu}_{2}^{+}$may be obtained by applying the state transition matrix between $t_{o}$ and $t_{2}$, and between $t_{2}$ and $t_{f}$. It is shown in reference 8 that the final mass is related to $\vec{r}_{2}$ and $t_{2}$ as follows: 


$$
\begin{aligned}
& \vec{\nabla}_{\vec{r}_{2}} m_{f}=\vec{\mu}_{2}^{+}-\vec{\mu}_{2}^{-} \\
& \frac{\partial m_{f}}{\partial \mathrm{t}_{2}}=-\left(\mathrm{H}_{2}^{+}-\mathrm{H}_{2}^{-}\right)
\end{aligned}
$$

The optimum values of $\vec{r}_{2}$ and $t_{2}$ (and corresponding values of spacecraft propellant mass and direction of impulse) may be determined by using a search procedure to maximize $m_{f}$ with respect to $\vec{r}_{2}$ and $t_{2}$, using the gradients in equation (B5). In this report, the Fletcher-Powell modified gradient method (ref. 4) was used to perform this optimization. 


\section{REFERENCES}

1. Gobetz, F. W.; and Doll, J. R. : How to Open the Heliocentric Launch Window for Earth-Mars Orbiter Missions. J. Spacecraft Rockets, vol. 6, no. 4, Apr. 1969, pp. 353-360.

2. Fimple, W. R. : Optimum Midcourse Plane Changes for Ballistic Interplanetary Trajectories. AIAA J., vol. 1, no. 2, Feb. 1963, pp. 430-434.

3. Doll, John R.; and Gobetz, Frank W.: Three-Impulse Interplanetary Rendezvous Trajectories. Proceedings of the Southeastern Symposium on Missiles and Aerospace Vehicle Sciences. Vol. 2. Am. Astron. Soc., 1966, pp. 55-1 to 55-14.

4. Fletcher, R.; and Powell, M. J. D. : A Rapidly Convergent Descent Method for Minimization. Computer J., vol. 6, 1963, pp. 163-168.

5. Escobal, Pedro R.: Methods of Orbit Determination. John Wiley \& Sons, Inc., 1965.

6. Pines, Samuel: Constants of the Motion for Optimum Thrust Trajectories in a Central Force Field. AIAA J., vol. 2, no. 11, Nov. 1964, pp. 2010-2014.

7. Danby, J. M. A. : The Matrizant of Keplerian Motion. AIAA J., vol. 2, no. 1, Jan. 1964, pp. 16-19.

8. Jezewski, D. J.; and Rozendaal, H. L.: An Efficient Method for Calculating Optimal Free-Space N-Impules Trajectories. AIAA J., vol. 6, no. 11, Nov. 1968, pp. 2160-2165. 


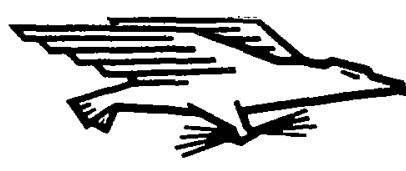

POSTAGE AND FEES PAID NATIONAL AERONAUTICS AND SPACE ADMINISTRATION

OSC 0OL 55 51305 7013400903

AIR FORCE WEAPQUS LABORATORY /WLOL/

KIRTLAND AFE, NEW MEXICO 87117

ATT E. LUU BOWNAN, CHIEF,TECH. LIBRARY

"The aeronatical and space activities of the United States sball be conducted so as to contribute ... to the expansion of buman knowledge of phenomena in the atmosphere and space. The Administration shall provide for the widest practicable and appropriate dissemination of information concerning its activities and the results thereof."

- National Aeronautics and Space ACt of 1958

\section{NASA SCIENTIFIC AND TECHNICAL PUBLICATIONS}

TECHNICAL REPORTS: Scientific and technical information considered important, complete, and a lasting contribution to existing knowledge.

TECHNICAL NOTES: Information less broad in scope but nevertheless of importance as a contribution to existing knowledge.

TECHNICAL MEMORANDUMS:

Information receiving limited distribution because of preliminary data, security classification, or other reasons.

CONTRACTOR REPORTS: Scientific and technical information generated under a NASA contract or grant and considered an important contribution to existing knowledge.
TECHNICAL TRANSLATIONS: Information published in a foreign language considered to merit NASA distribution in English.

SPECIAL PUBLICATIONS: Information derived from or of value to NASA activities. Publications include conference proceedings, monographs, data compilations, handbooks, sourcebooks, and special bibliographies.

\section{TECHNOLOGY UTILIZATION}

PUBLICATIONS: Information on technology used by NASA that may be of particular interest in commercial and other non-aerospace applications. Publications include Tech Briefs, Technology Utilization Reports and Notes, and Technology Surveys.

Details on the availability of these publications may be obtained from: 